\title{
As relações entre perfil de jogador e personalidade: um comparativo entre pesquisas.
}

\author{
The relations between player profile and personality: a comparative between \\ researches
}

MOREIRA, Victor; Departamento de Pós-Graduação em Design; Universidade Federal do Paraná victoremmoreira@gmail.com

OKIMOTO, Maria Lúcia; Departamento de Engenharia Mecânica; Universidade Federal do Paraná

lucia.demec@ufpr.br

\section{Resumo}

Para compreender melhor os desejos dos usuários, pesquisadores desenvolvem perfis que sintetizam as preferências de um determinado grupo. A taxonomia de jogadores fornece ao designer uma série de descrições de perfil que são úteis para o desenvolvimento e aprimoramento de jogos. No entanto, se o objetivo for estimular certas emoções em um público, o designer precisa também ter a percepção da personalidade emocional do consumidor. Por isso, o objetivo deste artigo é explorar as relações entre perfis de jogadores e perfis de personalidade. A partir da revisão bibliográfica sistemática listamos os principais trabalhos que relacionam estes dois temas. Discutimos os achados quanto a procedimentos metodológicos, modelos de perfil de jogador, modelos de personalidade emocional e, por fim, sintetizamos das relações encontradas nos resultados das pesquisas.

Palavras Chave: perfis de jogadores; perfis de personalidade; modelos; design emocional.

\begin{abstract}
To have a better understanding about the users' desires, researchers develop profiles that synthesize the preferences of a particular group. The player taxonomy provides the designer a series of profile descriptions that are useful for game development and enhancement. However, if the aim is to stimulate certain kind of emotion in an audience, the designer need to have also the perception of the consumer's emotional personality. Therefore, the purpose of this article is to explore the relation between player profiles and personality profiles. From the systematic bibliographical review, we list the main works that relate the two themes. We discuss the findings regarding: methodological procedures, player profile models, emotional personality models and, in the end, we synthesize the relation found in the results.
\end{abstract}

Keywords: player profiles; personality profiles; models; emotional design. 


\section{Introdução}

Lembra do fantasma perseguindo você em Super Mario World? E das corridas na chuva de Top Gear? E dos combates em The Legend of Zelda? E os sustos que levou ao enfrentar os zumbis de Resident Evil? Das loucuras que pode fazer em GTA? E das escolhas que fez em Mass Effect? Alguns dos momentos mais memoráveis nos jogos tem um forte impacto emocional sobre nós, isso acontece por que é da natureza humana se colocar no lugar do outro. O design emocional busca explicar o porquê sentimos certas emoções. Existem diversas teorias sobre os processos emocionais (MOORS, 2009), mas nesta pesquisa adotamos a Teoria dos Appraisals, na qual as emoções correspondem a um resultado combinado das preferências do indivíduo (concerns) diante de um estímulo (artefato). Esta combinação provoca um processo de avaliação (appraisal) no qual o produto final é a emoção do indivíduo (Figura 1).

Figura 1- Modelo básico de compreensão sobre emoções em relação a produtos.

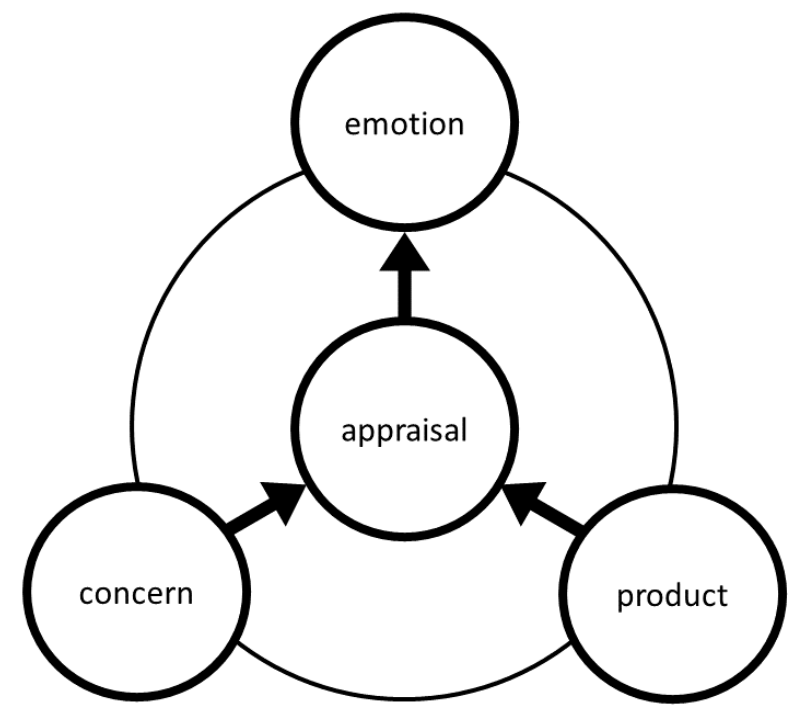

Fonte: Desmet (2002).

Demir, Desmet e Hekkert (2009) explicam que o design emocional tem como objetivo projetar com a intenção de evocar ou prevenir uma determinada emoção. Por tanto, não se pretende manipular ou induzir uma emoção no consumidor (o que provocaria conflitos éticos), mas sim conhecer e entender as características do consumidor a fim de promover uma combinação de preferências. A nível projetual, o design emocional pode apresentar-se de quatro formas: com foco no usuário, com foco no designer, com foco na pesquisa e com foco na teoria. Cada um destes focos diz respeito ao objetivo final do estudo, por exemplo, o foco no usuário é envolvido no projeto e suas emoções são o foco do processo de design; enquanto que, com foco na pesquisa, as diretrizes são o resultado da pesquisa (e.g entrevistas com usuários) (DESMET; HEKKERT, 2009).

A personalidade do usuário tem um papel importante na interação com o produto. Pervin e John (2004) explicam que a personalidade de um indivíduo é representada pelo conjunto de características que explicam padrões como sentimentos, pensamentos e comportamentos. Por isso a capacidade de entender e classificar estes padrões podem contribuir no desenvolvimento de um produto mais afetivo. Relacionar personalidade emocional para gerar diretrizes para o projeto é um 
campo recentemente explorado. Govers e Mugge (2004); Helfenstein (2012); Karsvall (2002) e Campelo, Scaletsky e Tonetto (2012) são exemplos de pesquisa que investigam formas de atrelar a personalidade dos usuários com características dos produtos.

A categorização de perfis de usuários é uma ferramenta comumente usada para sintetizar grupos de consumidores. Desmet, Overbeeke e Tax (2001) usam essa técnica para categorizar os consumidores conforme as preferências, e relacionam as personas com as emoções que cada produto induz. Na área de jogos existem pesquisas em busca de uma categorização dos perfis de jogadores, e o principal questionamento apresentado é sobre "Por que as pessoas jogam?". Na década de 90, Richard Bartle fez uma pesquisa com jogadores de MUD (Multi-user dungeon) a fim de encontrar padrões de motivação. $O$ autor descreve os 4 perfis de jogadores que encontrou (Killers, Achivers, Socialisers e Explorers) (BARTLE, 1996), este estudo é considerado, por diversos autores, como o trabalho sobre perfis de jogadores mais antigo e popular. O autor continuou aprimorando o modelo (BARTLE, 2003, 2005) buscando enquadrar o perfil dos jogadores sob a lente das novas tecnologias de jogos. Desde então outros autores também desenvolveram pesquisas sobre perfil de jogadores, dentre os quais destaca-se o de Yee (2007) que traçou o perfil dos jogadores de MMORPG (Massive Multiplayer Online Role-Playing Game). E Nacke, Bateman e Mandryk (2014) que utilizam dados neurobiológicos e demográficos para categorizar sete perfis de jogadores.

Nesse sentido é possível perceber a ligação entre o perfil do jogador e o perfil emocional. Dessa forma será possível aferir que um determinado perfil de jogador ao jogar um certo tipo de jogo irá experimentar uma certa gama de emoções. Se esta hipótese estiver correta, será possível listar uma série de diretrizes projetuais com base nos modelos teóricos já existentes. Portanto, buscamos apresentar uma revisão bibliográfica sistemática afim de verificar a existência de pesquisas nesta área. Neste artigo apresentamos e analisamos profundamente os principais achados da revisão e discutimos as suas relações.

\section{Método}

Para Gil (2008) a pesquisa bibliográfica tem uma natureza básica que procura responder as perguntas por meio de conceitos teóricos, podendo usar uma abordagem quantitativa ou qualitativa para revisar a literatura. As pesquisas bibliográficas, em sua maioria, têm objetivos descritivos e por isso privilegiam a abordagem qualitativa. A revisão bibliográfica pode ser classificada como: narrativa ou sistemática, sendo que a revisão bibliográfica narrativa é baseada em uma descrição simplificada de estudos e informações sobre um determinado assunto, enquanto que a sistemática aplica métodos com maior rigor científico podendo alcançar melhores resultados (COOK; MULROW; HAYNES, 1997).

Nesse sentido, utilizamos os processos de revisão bibliográfica sistemática descritas por Conforto, Amaral e Silva (2011). Por tanto, o nosso primeiro passo foi listar as palavras-chave da busca e, em seguida, selecionar nove bases de dados científicos. Por fim, definimos critérios de seleção como, por exemplo, artigos em português e inglês. Os dados estatísticos sobre este levantamento de dados são descritos em profundidade por (omitido para revisão cega).

\section{Resultados}

A partir da análise dos resultados (MOREIRA; ULBRICHT, 2017) verificou-se que três trabalhos que estão mais próximos do foco da pesquisa. Os trabalhos de Graham e Gosling (2013); 
Paulin (2013); Zeigler-hill e Monica (2015) buscam relacionar o perfil do jogador com o perfil emocional e, por tanto, estas pesquisas serão analisadas profundamente. Dividimos a análise em quatro pontos principais: procedimentos metodológicos, modelos de perfil de jogador, modelos de personalidade emocional e resumo das relações entre os resultados das pesquisas.

\subsection{Procedimentos Metodológicos}

Os trabalhos de Graham e Gosling (2013); Zeigler-hill e Monica (2015) enquadram-se no tipo de pesquisa aplicada pois ambos utilizam questionários para coletar dados dos jogadores. No primeiro os autores utilizam o modelo de motivação de jogadores de YEE (2007) para coletar informações sobre o perfil dos jogadores de MMORPG. E para avaliar o perfil emocional utilizam o modelo Big Five de John e Srivastava (1999). Já no segundo os autores usam o modelo BrainHex de Nacke, Bateman e Mandryk (2014) para coletar o perfil dos jogadores de qualquer tipo de jogo. E usam o modelo HEXAGO de Ashton e Lee (2009) para avaliar o perfil emocional. É importante notar que os públicos destes dois trabalhos são diferentes e a quantidade de questionários respondidos também. No primeiro os autores analisam as respostas de 1.413 jogadores, enquanto que o segundo analisa as respostas de 359 jogadores.

Já o terceiro trabalho (PAULIN, 2013) enquadra-se no tipo de pesquisa básica pois relaciona conceitos de vários modelos buscando por semelhanças, ativações e princípios. Na dissertação de Paulin (2013) e no artigo de Paulin, Battaiola e Alves (2014), o autor relaciona o perfil BrainHex (NACKE; BATEMAN; MANDRYK, 2014), com as caraterísticas e preferências em jogos (BULHÕES; ALVES; DUBIELA, 2010), com os tipos psicológicos (MYERS; MYERS, 1997), com as chaves emocionais (LAZZARO, 2004), com as emoções correspondentes (BARTLE et al., 2009) e os aspectos correspondentes em jogos (ERMI; MÄYRÄ, 2005). Por conta da complexidade deste trabalho optamos em relacionar apenas perfil do jogador BrainHex as chaves emocionais de Lazzaro (2004).

No Quadro 1 listamos os modelos utilizados nas pesquisas citadas. É possível perceber que o modelo BrainHex aparece em duas das três pesquisas, sendo usado em pesquisa teórica e prática.

Quadro 1 - Definição e critérios de avaliação do artigo completo

\begin{tabular}{lll} 
Artigo & Modelo & Autor do modelo \\
\hline Graham e Gosling (2013) & $\begin{array}{l}\text { Motivações de jogadores } \\
\text { Big Five }\end{array}$ & $\begin{array}{l}\text { (YEE, 2007) } \\
\text { (JOHN; SRIVASTAVA, 1999) }\end{array}$ \\
\hline $\begin{array}{l}\text { (ZEIGLER-HILL; MONICA, } \\
2015)\end{array}$ & BrainHex & $\begin{array}{l}\text { (NACKE; BATEMAN; } \\
\text { HANDRYK, 2014) }\end{array}$ \\
\hline (PAULIN, 2013; PAULIN; & BrainHex & (ASHTON; LEE, 2009) \\
BATTAIOLA; ALVES, 2014) & Four keys to fun & (NACKE; BATEMAN; \\
\hline
\end{tabular}

Fonte: Elaborado pelo autor.

\subsection{Modelos de perfil de jogador}

No modelo de perfil de jogadores BrainHex (NACKE; BATEMAN; MANDRYK, 2014) os autores identificam sete tipos de preferências entre jogadores. O perfil Seeker gosta de explorar coisas e descobrir seus arredores; O perfil Survivor desfruta de cenas assustadoras associadas a fortes emoções em escapar de situações aterrorizantes; O perfil Daredevil desfruta da emoção de correr 
riscos; O perfil Mastermind desfruta de resolver quebra-cabeças, tomada de decisões difíceis e criar estratégias; O perfil Conqueror gosta de lutar para superar desafios difíceis; O perfil Socializer busca por interação com outros jogadores; O perfil Achiever desfruta de completar tarefas e atingir metas. Este perfil de jogadores conhecido como BrainHex tem mais de 50 mil respostas, é possível acessar pelo site blog.brainhex.com e o questionário (em inglês) está disponível no site survey.ihobo.com/BrainHex.

No entanto é importante examinar o contexto do desenvolvimento do modelo Brainhex. Nacke, Bateman e Mandryk (2014) baseiam-se em diversos modelos de perfis de jogadores como Bartle (1996) e Yee (2006), porém o modelo de personalidade dos jogadores é baseado no MyersBriggs Type Indicator (MBTI). A tipologia psicológica de Myers et al. (1985) divide em 16 tipos de personalidades que são determinadas por meio de quatro dimensões básicas onde cada um constitui uma preferência oposta, ou seja, a Extraversion é oposta a Introversion, Sensing é oposta a Intuition e assim sucessivamente. Contudo existem diversas pesquisas que entram em desacordo com esse modelo (McCrae e Costa, 1989; Murray, 1990 e Pittenger, 1993), a principal crítica ao modelo MBTI é por não cumprir com os padrões de testes psicológicos válidos. Porém o uso dessa tipologia no modelo de Nacke, Bateman e Mandryk (2014) não invalida os resultados obtidos.

Yee (2002) baseia-se nos conceitos de Bartle (1996) para desenvolver a sua própria taxonomia. Começando com estudos empíricos para avaliar as motivações de jogadores de MMORPG. Inicialmente elegendo cinco fatores motivacionais (Relationship, Immersion, Grief, Achievement e Leadership) (Yee, 2002), e mais tarde resumindo para três fatores principais (Achievement, Social e Immersion) e mais dez subfatores (Figura 2) (Advancement, Mechanics, Competition, Socializing, Relationship, Teamwork, Discovery, Role-Playing, Customization e Escapism) (Yee, 2007).

Figura 2: Modelo de motivações de jogadores de MMORPG.

\begin{tabular}{|c|c|c|}
\hline Achievement & Social & Immersion \\
\hline Advancement & Socializing & Discovery \\
Progress, Power, & Casual Chat, Helping Others, & Exploration, Lore, \\
Accumulation, Status & Making Friends & Finding Hidden Things \\
\hline Mechanics & Relationship & Role-Playing \\
Numbers, Optimization, & Personal, Self-Disclosure, & Story Line, Character History, \\
Templating, Analysis & Find and Give Support & Roles, Fantasy \\
\hline Competition & Teamwork & Customization \\
Challenging Others, & Collaboration, Groups, & Appearances, Accessories, \\
Provocation, Domination & Group Achievements & Style, Color Schemes \\
\hline & & Escapism \\
\hline & & Relax, Escape from RL, \\
& & Avoid RL Problems \\
\hline
\end{tabular}

Fonte: Yee (2007)

Alguns dos principais modelos de perfis de jogadores podem se correlacionar. Hamari e Tuunanen (2015) listam os modelos de perfis de jogadores e encontram várias correlações entre os conceitos utilizados pelos autores. Por exemplo, o conceito de perfil Achivement é encontrado em 10 dos 12 modelos e o conceito de perfil Sociability é encontrado em 9 dos 12 modelos. Em linhas 
gerais, é possível perceber que o mesmo conceito de perfil de jogador se repete em várias pesquisas (Figura 3).

Figura 3: Relações entre conceitos de perfis de jogadores

\begin{tabular}{|c|c|c|c|c|c|c|c|c|c|c|c|c|}
\hline Concept & 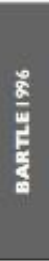 & 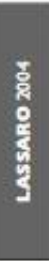 & 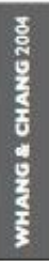 & 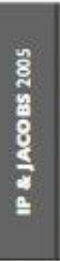 & 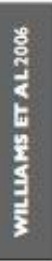 & 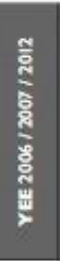 & 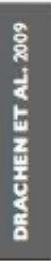 & 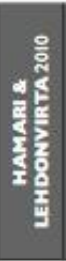 & 을 & 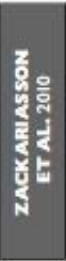 & 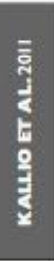 & है \\
\hline $\begin{array}{l}\text { Gaming intensity and skill (Hardcore, } \\
\text { Committed mentalities, Aggressive gamer, Veteran, } \\
\text { Casual, Cosual mentalities, Inactive gamer, Pocifist, } \\
\text { Avator level,Amateur) }\end{array}$ & & & & $\checkmark$ & & & $\checkmark$ & $\checkmark$ & $\checkmark$ & & $\checkmark$ & \\
\hline $\begin{array}{l}\text { Achievement (Achiever, Single-oriented player, } \\
\text { Guordion/Achiever,Aggressive gomer,Achievement, } \\
\text { Progress \& provocation, Power \& domination, Runner, } \\
\text { hord fun, Cosual (Stewart), Avotor level, (Semi-) } \\
\text { professional) }\end{array}$ & $\checkmark$ & $\checkmark$ & $\checkmark$ & & $\checkmark$ & $\checkmark$ & $\checkmark$ & $\checkmark$ & $\checkmark$ & $\checkmark$ & & $\checkmark$ \\
\hline $\begin{array}{l}\text { Exploration (Explorer, Solver, RotionallExplorer, } \\
\text { Azeressive gamer, Social gamer, Immersion. } \\
\text { Exploration \& Fontosy, Story \& escopism, curiosity) }\end{array}$ & $\checkmark$ & $\checkmark$ & & & & $\checkmark *$ & $\checkmark$ & & $\checkmark$ & $\checkmark$ & & $\checkmark$ \\
\hline $\begin{array}{l}\text { Sociability (Socialiser, Social mentalties, } \\
\text { Community-oriented player, Idealist/Socialiser, Social, } \\
\text { Helping \& support, the people foctor, Friends \& } \\
\text { Collaboration, (Semi-)professional,Amateur) }\end{array}$ & $\checkmark$ & $\checkmark$ & $\checkmark$ & & $\checkmark$ & $\checkmark$ & & & $\checkmark$ & $\checkmark$ & $\checkmark$ & $\checkmark$ \\
\hline $\begin{array}{l}\text { Domination (Killer,Artison/Killer, Aggressive gamer, } \\
\text { off-real world oriented player, Progress \& provocation, } \\
\text { Power \& domination, Cosual (Stewart)) }\end{array}$ & $\checkmark$ & & $\checkmark$ & & & & & & $\checkmark$ & $\checkmark$ & & $\checkmark$ \\
\hline $\begin{array}{l}\text { Immersion (Immersion, Committed mentolities, } \\
\text { Exploration \& fontosy, Story \& escopism, Off-real } \\
\text { world oriented player, altered stotes, Hardcore } \\
\text { (Stewort)) }\end{array}$ & & $\checkmark$ & $\checkmark$ & & $\checkmark$ & $\checkmark$ & & & & $\checkmark$ & $\checkmark$ & $\checkmark$ \\
\hline $\begin{array}{l}\text { In-game demographics (Avotar class ond } \\
\text { profession, non-dan member, Amateur, (Semi-) } \\
\text { professional, Group centrality. Size of the guild, Type } \\
\text { of server, Foction) }\end{array}$ & & & & & $\checkmark$ & & & $\checkmark$ & & & & \\
\hline
\end{tabular}

Fonte: Hamari e Tuunanen (2015)

\subsection{Modelos de perfil de emocional}

O modelo emocional de Niclole Lazzaro conhecido como Four Keys to Fun foi desenvolvido tomando como base três diretrizes: identificar/focar e entender o que os jogadores mais gostam nos jogos; serem mensuráveis por pesquisadores e serem controláveis por designers (LAZZARO, 2004). Foram coletados dados de observação das expressões faciais de 30 jogadores, totalizando 2.000 observações gravadas em vídeo. Analisando momentos dos jogos que os jogadores gostaram mais, ou seja, momentos que causam reações faciais. A autora dividiu em quatro grandes grupos:

- Hard Fun: A oportunidade de desafio e desenvolvimento da maestria;

- Easy Fun: A inspiração da imaginação e da brincadeira;

- Serious Fun: Um passaporte para o relaxamento e para ficar mais esperto ou mudar a si mesmo; 
- People Fun: Uma desculpa para encontrar e se relacionar com amigos.

O modelo de (Lazzaro, 2004) é utilizado como referência para explicar o perfil BrainHex. No Quadro 2 listamos as relações que Nacke, Bateman e Mandryk (2014) fazem com o modelo de Lazzaro (2004) e de Yee (2006).

Quadro 2: A relações entre taxonomias

\begin{tabular}{|l|l|l|}
\hline $\begin{array}{l}\text { Perfil BrainHex (NACKE; BATEMAN; } \\
\text { MANDRYK, 2014) }\end{array}$ & $\begin{array}{l}\text { Quatro chaves emocionais (LAZZARO, } \\
\text { 2004) }\end{array}$ & $\begin{array}{l}\text { Motivações de jogadores de } \\
\text { MMORPG (YEE, 2006) }\end{array}$ \\
\hline Seeker & Easy Fun & Immersion \\
\hline Survivor & Serious Fun & - \\
\hline Daredevil & Serious Fun & - \\
\hline Mastermind & Hard Fun & Manipulaton Motivation \\
\hline Conqueror & Hard Fun & - \\
\hline Socialiser & People Fun & Relationship Motivation \\
\hline Achiever & - & Achievement \\
\hline
\end{tabular}

Fonte: Formulado pelo autor com base no artigo de Nacke, Bateman e Mandryk (2014)

O modelo de personalidade Big Five de John e Srivastava (1999) é dividido em cinco grandes características: extraversion, agreeableness, conscientiousness, neuroticism, e openness. Yee et al. (2011) explicam que essas características podem ser associadas a comportamentos de jogadores, por exemplo, extrovertidos (extraversion) tendem a ser motivados por trabalho em equipe, enquanto que abertura a experiências (openness) está positivamente associada com a motivação para escapar da realidade. Yee (2016) defende que os jogos que jogamos são um reflexo e não uma fuga e estão mais relacionados com a própria identidade do indivíduo. Por isso a mensuração de personalidade emocional dos jogadores está ligada as ações e decisões do jogador no jogo.

Já o modelo HEXACO de Ashton e Lee (2009) é composto por seis fatores que inclui cinco variantes de personalidade (Honesty-Humility, Emotionality, extroversion, Agreeableness, Conscientiousness, Openness to Experience). Esse modelo capta o grau em que os indivíduos são suscetíveis a estados emocionais negativos (e.g preocupação, ansiedade). Por exemplo, a Extroversão (eXtraversion) reflete as características como sociabilidade, dominância e eloquência, enquanto que Conscienciosidade (Conscientiousness) é caracterizada por atributos como: cuidado, auto-disciplina e fiabilidade. Três das dimensões do modelo HEXACO (extraversion, conscientiousness e openness) se assemelham igualitariamente ao Big Five, e a Emotionality é equivalente a Neuroticism do modelo Big Five. Já a Agreeableness do HEXACO reflete levemente a Agreeableness do modelo Big Five.

Os modelos Big Five e HEXACO dispõem de questionários nos quais é possível coletar dados de um grande número de jogadores e fazer um levantamento quantitativo. Já o modelo Four Keys to Fun é baseado na observação das exceções faciais dos jogadores, favorecendo assim uma análise qualitativa dos jogadores.

\subsection{Síntese}

No Quadro 3 apresentamos um resumo dos resultados dos 3 artigos foco desta pesquisa. Nas colunas em verde estão os modelos de perfil emocional, e nas colunas em azul os modelos de perfil de jogador. Como a pesquisa de Paulin (2013) e Zeigler-hill e Monica (2015) utilizam o perfil 
Brainhex, estas estão sobre postas. Os perfis Achiever e Socializer estão presentes nos modelos BrainHex e de Yee (2007), e tomando como base estes dois perfis, relacionamos os demais conforme a descrição dos autores. No entanto, alguns dos perfis de Yee (2007) não poderão ser relacionados por falta de estudos que comprovem a relação com o perfil BrainHex.

Quadro 3: Relação entre o resultado das pesquisas.

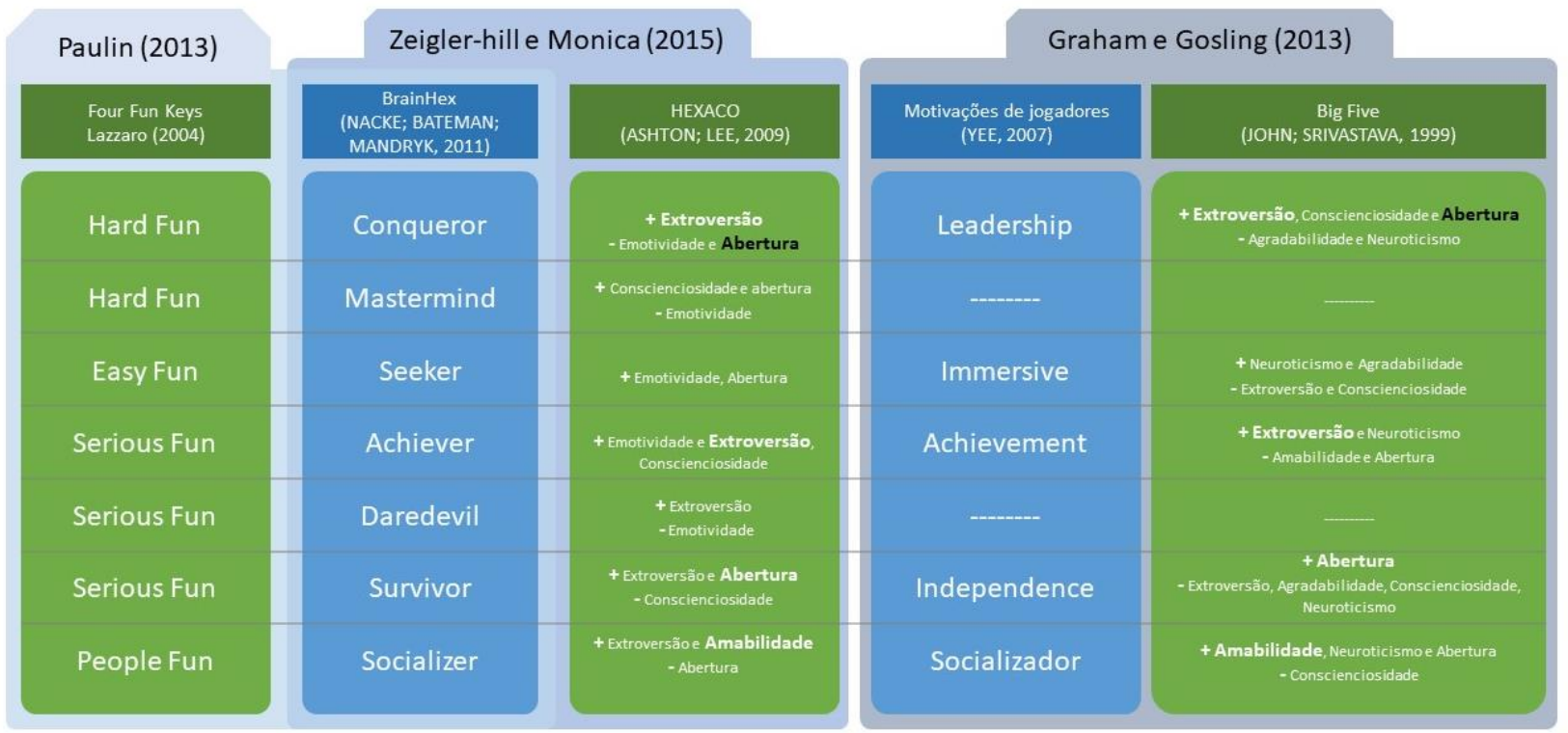

Fonte: Elaborado pelo Autor.

O primeiro ponto importante a se verificar é a relação dos resultados de Paulin (2013) com o de Nacke, Bateman e Mandryk (2014) (citado anteriormente no Quadro 2). Paulin (2013) realiza sua pesquisa baseado nos dados de Nacke, Bateman e Mandryk (2011), nesse artigo os autores não relacionam o perfil dos jogadores com o modelo de Lazzaro (2004). Por tanto, um ano antes Paulin (2013) já havia feito a relação que seria publicada posteriormente pelos autores do perfil BrainHex.

O segundo ponto diz respeito aos resultados do perfil emocional de Graham e Gosling (2013); Zeigler-hill e Monica (2015) que demonstram pouca similaridade na comparação. Colocamos em negrito branco os resultados similares e, em preto, os resultando contraditórios. Por exemplo, para Zeigler-hill e Monica (2015) o perfil Achiever está relacionado positivamente (+) com Emotividade e Extroversão e negativamente (-) com Conscienciosidade, e para Graham e Gosling (2013) o perfil Achievement está relacionado positivamente com Extroversão e Neuroticismo e negativamente com Amabilidade e Abertura. Ou seja, o único ponto em comum é a Extroversão. Encontramos também uma contradição ao relacionar o perfil Conqueror com Leadership, onde em um aparece negativamente relacionada com a Abertura e, no outro, positivamente.

\section{Conclusão}

Neste artigo analisamos profundamente os resultados da revisão sistemática de Moreira e Ulbricht (2017). Verificamos que relacionar o perfil do jogador com o a personalidade emocional fornece informações a nível projetual e de pesquisa. Tomando como base o modelo de compreensão sobre emoções em relação a produtos (DESMET, 2002), podemos traçar perfis de consumidores que buscam por uma gama de emoções. Para tanto, traçamos um recorte sobre a 
área de design de jogos baseando em teoria e modelos de perfis de jogadores e de personalidade. Nesse sentido a revisão sistemática de literatura resultou em três artigos que relacionam os dois tópicos.

Analisando o resultados das pesquisas de Graham e Gosling (2013); Paulin (2013); Zeiglerhill e Monica (2015) encontramos uma pesquisa de natureza teórica e duas com natureza prática. Verificamos que o perfil BrainHex de Nacke, Bateman e Mandryk (2014) foi utilizado em duas pesquisas. Encontramos divergências e similaridades ao relacionar o resultado dos estudos. No entanto, e se levarmos em consideração somente as similaridades, é possível traçar um paralelo consistente. No Quadro 4 formatamos apenas os pontos de similaridade entre os estudos, tornando possível fazer inferências entre os perfis, tais como: os perfis Conqueror, Leadership e Acheiver são atraídos pela Extroverssão, os perfis Survivor e Independence são atraídos pela Abertura e, o perfil Socializer, pela Amabilidade. No entanto é importante frisar que essas relações não são restringentes a seus perfis, mas sim características recorrentes nestes perfis.

Quadro 4: Apenas resultados similares entre as pesquisas.

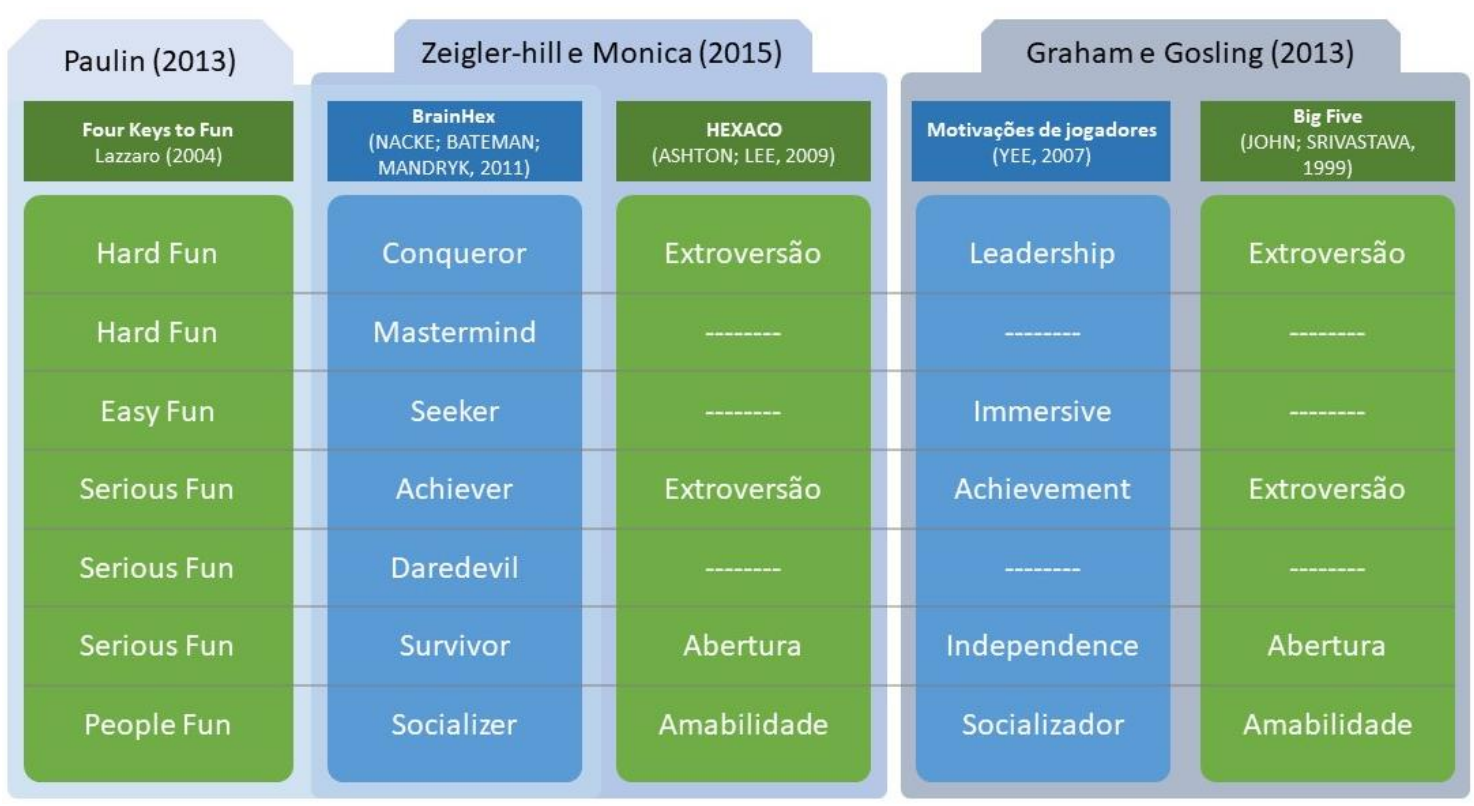

Fonte: Elaborado pelo autor.

Contudo, os resultados das pesquisas que relacionam o perfil do jogador com o perfil emocional até hoje, não apresentam resultados consistentes. Apoiamos esta afirmação nos resultados dispares e pouco recorrentes que encontramos. Considerando a amostra, técnicas de coleta e análise de dados, os resultados deveriam ter mais similaridade. Por tanto, listamos algumas possíveis formas de solucionar essa incongruência: aumentar o rigor na coleta de dados, embasar os dados com uma terceira fonte como por exemplo observação e agrupar populações de jogadores, como por exemplo, jogadores de MMORPG e de MOBA (Multiplayer Online Battle Arena).

\section{Referências}

ASHTON, M. C.; LEE, K. The HEXACO-60: A short measure of the major dimensions of personality. Journal of Personality Assessment, v. 91, n. 4, p. 340-345, 2009. 
BARTLE, R. Players Who Suit MUDs. Journal of MUD research, p. 1, 1996.

BARTLE, R. Designing Virtual Worlds. Indianapolis - USA: New Riders Publishing, 2003. v. 1

BARTLE, R. Virtual Worlds: Why People Play. Massively Multiplayer Game Development, v. 2, p. 318, 2005.

BARTLE, R. et al. Beyond game design: Nine steps toward creating better videogames. [s.I.] Course Technology, 2009.

BULHÕES, B.; ALVES, T.; DUBIELA, R. Framework CDE: com foco na experiência do usuário de jogos digitais. IX Simpósio Brasileiro de Jogos e Entretenimento Digital - SBGames 2010, p. 50-59, 2010.

CAMPELO, F.; SCALETSKY, C. C.; TONETTO, L. M. Designing for experience and public transportation : an emotional-driven project. Cumulus Conference 2012, n. 2000, 2012.

CONFORTO, E. C.; AMARAL, D. C.; SILVA, S. L. DA. Roteiro para revisão bibliográfica sistemática: aplicação no desenvolvimento de produtos e gerenciamento de projetos. v. 8, n. 1998, p. 1-12, 2011.

COOK, D. J.; MULROW, C. D.; HAYNES, R. B. Systematic reviews: synthesis of best evidence for clinical decisions. Ann Intern Med, v. 126, n. 5, p. 376, 1997.

DEMIR, E.; DESMET, P.; HEKKERT, P. Appraisal Patterns of Emotions in Human-Product Interaction. International Journal of Design, v. 3, n. 2, p. 41-51, 2009.

DESMET, P. Designing Emotions. [s.I.] Delft University of Technolog, 2002.

DESMET, P.; HEKKERT, P. Special Issue Editorial: Design \& Emotion. International Journal of Design, v. 3, n. 2, p. 1-6, 2009.

DESMET, P.; OVERBEEKE, C. J.; TAX, S. J. E. T. Designing Products with Added Emotional Value: Development and Appllcation of an Approach for Research Through Design. The Design Journal, v. 4, n. 1, p. 32-47, 2001.

ERMI, L.; MÄYRÄ, F. Fundamental Components of the Gameplay Experience: Analysing Immersion. Changing Views: Worlds in Play, p. 15-27, 2005.

GIL, A. C. Métodos e técnicas de pesquisa social. $6^{\circ}$ ed. São Paulo - SP: Atlas, 2008.

GOVERS, P. C. M.; MUGGE, R. "I love my Jeep, because it " $s$ tough like me', The effect of productpersonality congruence on product attachment. Proceedings of the Fourth International Conference on Design and Emotion, v. 31, p. 1-15, 2004.

GRAHAM, L. T.; GOSLING, S. D. Personality Profiles Associated with Different Motivations for Playing World of Warcraft. Cyberpsychology, Behavior, and Social Networking, v. 16, n. 3, p. 189-193, 2013.

HAMARI, J.; TUUNANEN, J. Player Types: A Meta-synthesis. Transactions of the Digital Games Research Association (ToDiGRA), v. Vol. 1, No, n. March 2014, p. 29-53, 2015.

HELFENSTEIN, S. Increasingly emotional design for growingly pragmatic users? A report from Finland. Behaviour and Information Technology, v. 31, n. 2, p. 185-204, 2012.

JOHN, O. P.; SRIVASTAVA, S. The Big Five trait taxonomy: History, measurement, and theoretical perspectives. Handbook of personality: Theory and research, v. 2, n. 510, p. 102-138, 1999. 
KARSVALL, A. Personality preferences in graphical interface design. Proceedings of the second Nordic conference on ..., p. 217-218, 2002.

LAZZARO, N. Why We Play Games: Four Keys to More Emotion in Player Experiences. Proceedings of GDC, v. 306, p. 1-8, 2004.

MOORS, A. Theories of emotion causation: A review. [s.l: s.n.]. v. 23

MOREIRA, V.; ULBRICHT, V. R. O perfil do jogador e as emoções no jogo. Proceedings of SBGames 2017: Art \& Design Track, 2017.

MYERS, I. B.; MYERS, P. B. Ser humano é ser diferente : valorizando as pessoas por seus dons especiais. [s.I.] Editora Gente, 1997.

NACKE, L.; BATEMAN, C.; MANDRYK, R. BrainHex: Preliminary results from a neurobiological gamer typology survey. Lecture Notes in Computer Science (including subseries Lecture Notes in Artificial Intelligence and Lecture Notes in Bioinformatics), v. 6972 LNCS, p. 288-293, 2011.

NACKE, L.; BATEMAN, C.; MANDRYK, R. BrainHex: A neurobiological gamer typology survey. Entertainment Computing, v. 5, n. 1, p. 55-62, 2014.

PAULIN, R. E. Mapeamento das Relações entre Perfis de Jogadores, Tipos Psicológicos, Emoções e Componentes de Jogos Eletrônicos, 2013. Disponível em: <http://hdl.handle.net/1884/35646>

PAULIN, R. E.; BATTAIOLA, A. L.; ALVES, M. M. The study of the relations between the brainhex player profiles, MBTI psychological types and emotions as means to enhance user experience. Lecture Notes in Computer Science (including subseries Lecture Notes in Artificial Intelligence and Lecture Notes in Bioinformatics), v. 8518 LNCS, n. PART 2, p. 732-741, 2014.

PERVIN, L. A.; JOHN, O. P. Personalidade: teoria e pesquisa. $8^{\circ}$ ed. São Paulo: Artmed, 2004.

YEE, N. The Demographics, Motivations and Derived Experiences of Users of Massively Multi-User Online Graphical Environments. Online, v. 15, n. 3, p. 309-329, 2006.

YEE, N. Motivations of Play in Online Games. CyberPsychology and Behavior, v. 9, p. 772-775, 2007.

YEE, N. et al. Introverted elves \& conscientious gnomes: The expression of personality in world of warcraft. Proceedings of the 2011 ..., p. 753-762, 2011.

YEE, N. Gaming Motivations Align with Personality Traits Action-Social is an Expression of. Disponível em: <https://quanticfoundry.com/2016/01/05/personality-correlates/>. Acesso em: 5 fev. 2018.

ZEIGLER-HILL, V.; MONICA, S. The HEXACO model of personality and video game preferences. Entertainment Computing, v. 11, p. 21-26, 2015. 